\title{
Acquired onset of third, fourth, and sixth cranial nerve palsies in children and adolescents
}

\author{
Kyung-Ah Park ${ }^{1} \cdot$ Sei Yeul Oh ${ }^{1} \cdot$ Ju-Hong Min ${ }^{2} \cdot$ Byoung Joon $\mathrm{Kim}^{2} \cdot$ Yikyung Kim ${ }^{3}$
}

Received: 21 January 2018 / Revised: 10 September 2018 / Accepted: 17 October 2018 / Published online: 13 February 2019

(c) The Royal College of Ophthalmologists 2019

\begin{abstract}
Purpose To describe the causes of third, fourth, and sixth cranial nerve palsies in children and adolescents. Methods In this retrospective case series, a total of 66 patients aged 0-19 years with third, fourth, and sixth cranial nerve palsies seen in strabismus and neuro-ophthalmic practice from 2010 to 2017 were included. Causes of palsies were determined based on clinical assessment, high-resolution magnetic resonance imaging (MRI), and laboratory work-up. Results Thirty-five patients had sixth cranial nerve palsy, 14 patients had third cranial nerve palsy ( 7 partial, 7 complete), 13 patients had fourth cranial nerve palsy, and 4 patients had combined cranial nerve palsies in this study. Neoplasia involving central nervous system was one of the most common causes of third, fourth, and sixth cranial nerve palsies both in children (age: $0-14$ years) and adolescents (age: $15-19$ years) (20\% and $31 \%$, respectively). Overall, neoplasia (23\%) was the most common cause of acute third, fourth, and sixth cranial nerve palsies, followed by idiopathic cause (14\%), inflammation (11\%), and non-aneurysmal vascular contact (11\%). Neoplasia was also the most common cause of sixth and third cranial nerve palsies ( $25 \%$ and $29 \%$, respectively). The most common cause of fourth cranial nerve palsy was late decompensation in congenital fourth cranial nerve palsy $(46 \%)$.

Conclusions A substantial proportion of pediatric and juvenile patients had serious pathologies for third, fourth, and sixth cranial nerve palsies. If nerve palsies are indicated, prompt diagnosis of etiologies using high-resolution MRI with contrast and laboratory work-up are important for this disease population.
\end{abstract}

\section{Introduction}

Acquired isolated third, fourth, and sixth cranial nerve palsies are rarer entities in children and adolescents compared with those in adults. One population-based study has reported an estimated annual incidence of 7.6 per 100,000 [1]. Since the 1970s, several studies have reported causes of third, fourth, and sixth cranial nerve palsies in children [1-11]. Studies have generally agreed that acquired ocular motor nerve palsies in children could be regarded as

Sei Yeul Oh

syoh@skku.edu

1 Department of Ophthalmology, Samsung Medical Center, Sungkyunkwan University School of Medicine, Seoul, Korea

2 Department of Neurology, Samsung Medical Center, Sungkyunkwan University School of Medicine, Seoul, Korea

3 Department of Radiology, Samsung Medical Center, Sungkyunkwan University School of Medicine, Seoul, Korea ominous sign for serious pathologies. Regarding sixth cranial nerve palsy, neoplasia or trauma has been reported to be the leading cause in pediatric population $[2-4,6,7,10]$. The proportion of neoplasia involving central nervous system has been reported to be $19-45 \%$ in these studies. Such wide range of neoplasia might be owing to difference in study settings. In third cranial nerve palsy, trauma has been reported to be the most common acquired cause in pediatric population in most studies $[1,6,8,9,11]$. Neoplasia has been reported to be a cause of third cranial nerve palsy in $14-21 \%$ of pediatric patients $[1,6,8,9,11]$. In addition to various types of neoplasia, serious pathologies including meningitis, encephalitis, intracranial aneurysm, and poison intoxication have been reported to be causes of acquired third nerve palsy in previous studies $[1,6,8,9,11]$. For fourth cranial nerve palsies in pediatric population, the most common cause has also been reported to be trauma [4, 6]. Neoplasia has been reported to be a cause in $5 \%$ of pediatric patients [6]. The proportion of isolated cases and not-isolated cases combined with other neurologic signs has differed between studies. Inclusion criteria were also 
different between studies. Such differences might have affected the proportion of etiologies in each study.

However, many studies are published before the advent of modern neuroimaging technique that can display each cranial nerve. The development of high-resolution magnetic resonance imaging (MRI) has enabled us to detect pathologies not detected or diagnosed in the past $[12,13]$. Schwannoma of cranial nerves in its early stage and nonaneurysmal vascular contact $[14,15]$ are such examples. The use of three-dimensional steady-state free precession sequences and modified fully refocused steady-state sequences such as constructive interference in steady-state (CISS)/fast imaging employing steady-state acquisition cycled phases (FIESTA-C)/balanced fast field echo (B-FFE) has provided augmented visualization of cranial nerves in vivo.

Prompt and appropriate diagnostic work-up for patients with cranial nerve palsies is very important owing to potential of long-term loss of function and life-threatening condition. The objective of this study was to investigate causes of acquired onset of third, fourth, and sixth cranial nerve palsies in pediatric and juvenile patients based on clinical assessment, high-resolution MRI, and laboratory work-up.

\section{Methods}

A retrospective review was performed for pediatric and juvenile patients with acquired onset of third, fourth, and sixth cranial nerve palsies. They were followed-up at Strabismus and Neuro-ophthalmology Department of Samsung Medical Center from March 2010 to August 2017 according to tenets of the Declaration of Helsinki. This study was approved by the Institutional Review Board of Samsung Medical Center. Patients aged 0-19 years presenting acquired third, fourth, or sixth cranial nerve palsy were included. At the initial visit, all patients underwent a full ophthalmologic assessment, including visual acuity examination, evaluation of ocular alignment status, slit-lamp biomicroscopy, and fundus examination. Ocular alignment was determined by cover test, cover-uncover test, and prism alternate cover test at fixation of $6-\mathrm{m}$ and $33-\mathrm{cm}$. Voluntary ductions were performed for all patients.

MRI or computed tomography image obtained from outside hospital or other departments before referral was reviewed if present. High-resolution cranial nerve MRI with three-dimensional sequences was performed if the etiology could not be clearly revealed from previous brain images. MRI was performed using a 3-T system (Philips Achieva 3 T, Philips Medical Systems, Best, The Netherlands) for all patients with a 32-channel phased-array head coil. T2-weighted imaging was performed to visualize the entire brain. Axial T2-weighted imaging was performed using a turbo spin-echo sequence. Heavily T2-weighted images were obtained using a three-dimensional volume isotropic turbo spin-echo acquisition (VISTA) sequence to visualize the cisternal segment of the cranial nerves. Detailed parameters of 3D-VISTA sequence were as follows: repetition time, $2000 \mathrm{~ms}$; echo time, $242 \mathrm{~ms}$; flip angle, $90^{\circ}$; field of view $170 \times 170 \mathrm{~mm}$; matrix, $284 \times 284$; section thickness, $0.3 \mathrm{~mm}$; slab thickness, $5.4 \mathrm{~cm}$, and acquisition time, $9 \mathrm{~min}$ and $8 \mathrm{~s}$. Additional three-dimensional balanced turbo field echo sequence was performed to visualize cisternal segment of the fourth cranial nerve with the following parameters: repetition time, $8.2 \mathrm{~ms}$; echo time, $4.1 \mathrm{~ms}$; flip angle, $50^{\circ}$; field of view, $160 \times 160 \mathrm{~mm}$; matrix, $532 \times 533$; section thickness, $0.25 \mathrm{~mm}$, slab thickness, $1.75 \mathrm{~cm}$, and acquisition time, $4 \mathrm{~min}$ and $57 \mathrm{~s}$. After the intravenous injection of $0.1 \mathrm{mmol} / \mathrm{kg}$ of gadolinium based contrast material, threedimensional turbo field echo sequence with fat saturation was obtained, using following parameters: repetition time, $9.6 \mathrm{~ms}$; echo time, $3.18 \mathrm{~ms}$; flip angle, $8^{\circ}$; field of view, $180 \times 180 \mathrm{~mm}$; matrix, $272 \times 272$; section thickness, $0.5 \mathrm{~mm}$, slab thickness, $8 \mathrm{~cm}$, and acquisition time, $6 \mathrm{~min}$ and $23 \mathrm{~s}$.

The following tests were also performed when the etiology of cranial nerve palsy was unclear from previous neuroimaging: test for complete blood cell count, chemistry panel, serologic tests including syphilis serologic test, thyroid stimulating hormone receptor antibodies, acetylcholine receptor antibody, and ganglioside antibody panel. Edrophonium test, neostigmine test, and repetitive nerve stimulation test were considered for selected cases based on clinical presentation.

\section{Results}

Sixty-six pediatric and adolescent patients were included in this study (Table 1), including 50 patients aged $<15$ years and 16 patients aged $15-19$ years. Their mean age was $9 \pm 6$ years (range, 0-19 years). Forty-three (65\%) patients were boys and $23(35 \%)$ patients were girls. All patients were Asians. There were 23 right-sided palsies and 29 left-sided palsies. Fourteen patients had bilateral third, fourth, or sixth cranial nerve palsies. Nine of these bilateral cases were equally paretic on each side. Four cases had primarily rightsided palsies while one case had primarily left-sided palsy. There were 35 patients with a sixth cranial nerve palsy, 14 patients with a third cranial nerve palsy ( 7 partial and 7 complete), and 13 patients with a fourth cranial nerve palsy. Four patients had combined third, fourth, or sixth cranial nerve palsies. For 26 patients who presented to our clinic with previous neuroimaging results, etiologies of cranial nerve palsy could be identified with conventional 
Table 1 Characteristics and underlying etiologies in patients with acute third, fourth, and sixth cranial nerve palsies and comparisons among third, fourth, and sixth cranial nerve palsies

\begin{tabular}{|c|c|c|c|c|c|}
\hline & \multicolumn{5}{|c|}{ Third, fourth, and sixth cranial nerve palsies } \\
\hline & $\begin{array}{l}\text { Sixth } \\
(n=35)\end{array}$ & $\begin{array}{l}\text { Third } \\
(n=14)\end{array}$ & $\begin{array}{l}\text { Fourth } \\
(n=13)\end{array}$ & $\begin{array}{l}\text { Combined } \\
(n=4)\end{array}$ & Total \\
\hline Age (mean $\pm \mathrm{SD})$ (years) & $9 \pm 7$ & $7 \pm 7$ & $7 \pm 5$ & $14 \pm 1$ & $9 \pm 6$ \\
\hline Gender (male/female) & $19 / 17$ & $10 / 4$ & $10 / 3$ & $4 / 0$ & $43 / 23$ \\
\hline \multicolumn{6}{|l|}{ Underlying etiologies } \\
\hline Neoplastic & $9(25 \%)$ & $4(29 \%)$ & $2(15 \%)$ & & $15(23 \%)$ \\
\hline Gliomatous & 4 & & & & 4 \\
\hline Other primary & 2 & 2 & 2 & & 6 \\
\hline Metastatic or progression & 2 & 1 & & & 3 \\
\hline $\begin{array}{l}\text { After surgical resection of brain } \\
\text { tumor }\end{array}$ & 1 & 1 & & & 2 \\
\hline Inflammatory & $4(11 \%)$ & $3(21 \%)$ & & & $7(11 \%)$ \\
\hline Intracranial hemorrhage & $1(3 \%)$ & $1(7 \%)$ & $1(8 \%)$ & & $3(5 \%)$ \\
\hline Cavernous sinus thrombophlebitis & & & & $1(25 \%)$ & $1(2 \%)$ \\
\hline Vascular malformation & & $1(7 \%)$ & $1(8 \%)$ & & $2(3 \%)$ \\
\hline Hydrocephalus & $2(6 \%)$ & & & & $2(3 \%)$ \\
\hline Multiple sclerosis & $1(3 \%)$ & & & & $1(2 \%)$ \\
\hline $\begin{array}{l}\text { Chemotherapy induced } \\
\text { leukoencephalopathy }\end{array}$ & $1(3 \%)$ & & & & $1(2 \%)$ \\
\hline Non-aneurysmal vascular contact & $6(17 \%)$ & $1(7 \%)$ & & & $7(11 \%)$ \\
\hline Miller Fisher syndrome & $3(8 \%)$ & & & $3(75 \%)$ & $6(9 \%)$ \\
\hline Traumatic & $4(11 \%)$ & $1(7 \%)$ & $1(8 \%)$ & & $6(9 \%)$ \\
\hline Idiopathic & $4(11 \%)$ & $3(21 \%)$ & $2(15 \%)$ & & $9(14 \%)$ \\
\hline Congenital $^{\mathrm{a}}$ & & & $6(46 \%)$ & & $6(9 \%)$ \\
\hline
\end{tabular}

$\mathrm{SD}=$ standard deviation

${ }^{a}$ These patients had acquired onset. However, the etiology was concluded as congenital based on the absence or atrophy of fourth nerve and/or superior oblique muscle in the paretic eye
MRI and/or computed tomography. For the remaining 40 patients who did not have previous neuroimaging or in whom etiologies of cranial nerve palsies were not clearly revealed from previous brain images, high-resolution brain pre- and post-MRI with three-dimensional steady-state free precession sequences and modified fully refocused steadystate sequences (CISS) were performed. Tests for complete blood cell count, chemistry panel, serologic tests including syphilis serologic test, thyroid-stimulating hormone receptor antibodies, acetylcholine receptor antibody, and ganglioside antibody were also performed for a total of 41 patients. Edrophonium test, neostigmine test, and repetitive nerve stimulation test were performed for three cases based on their clinical presentations.

The number of patients in each etiologic category of acquired onset of third, fourth, and sixth cranial nerve palsies is shown in Table 1 .

\section{Sixth cranial nerve palsy}

Of 35 subjects with sixth cranial nerve palsy, neuroimaging and other studies identified neoplasia involving central nervous system in nine patients (including two patients with metastatic lesions from previously diagnosed malignancies and one patient who had surgical removal of brain tumor), inflammation in four patients, miller fisher syndrome in three patients, multiple sclerosis in one patient (in whom palsy was the only presenting sign of the demyelinating disease), hydrocephalus in two patients, intracranial hemorrhage in one patient, and chemotherapy induced leukoencephalopathy in one patient as etiologies for sixth cranial nerve palsies.

Among six patients with newly diagnosed primary brain tumor, four patients had glioma (Fig. 1). One patient with negative conventional MRI result was found to have neoplasia when repeated high-resolution MRI was performed. Of two patients with metastatic brain tumor, one patient (19 years old) had been in complete remission for 14 years after chemotherapy, radiotherapy, and subsequent hematopoietic stem cell transplantation for neuroblastoma before abrupt development of isolated sixth cranial nerve palsy. After the diagnosis of metastasis was made, the patient received surgical resection of metastatic lesions. The patient has been in complete remission for 


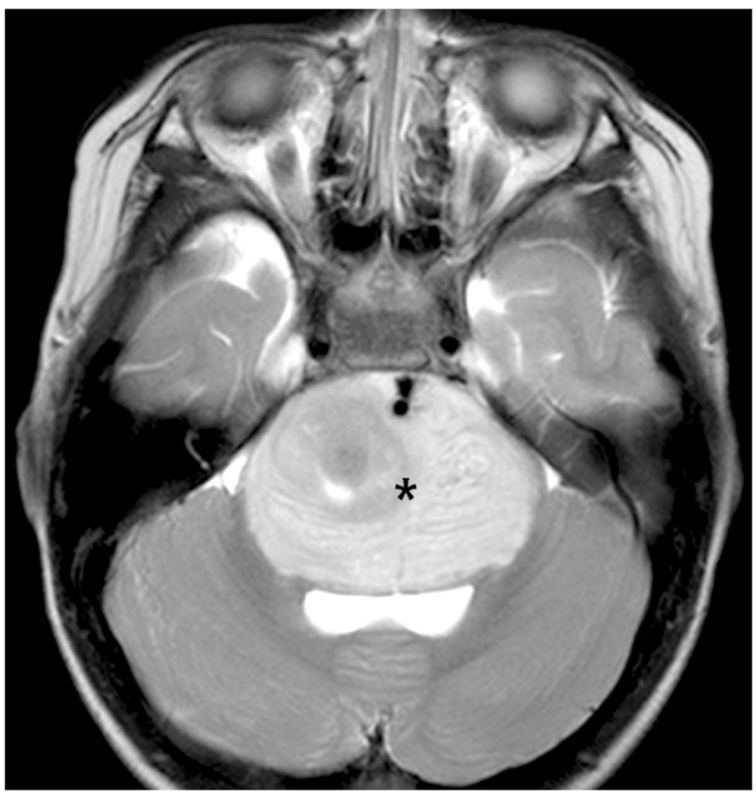

Fig. 1 An infiltrative mass lesion causing bilateral sixth nerve palsy (asterisk). A 3-year-old girl presented with intermittent head turn to the right side for 3 months and mild esodeviation for 3 weeks. The patient's guardian reported that the deviation had been improved continuously before presentation. The patient had esodeviation of 12 prism diopters and mild abduction limitation in both eyes. Immediate magnetic resonance imaging was performed. Although the patient was found to have large infiltrative mass involving brainstem and cerebellar parenchyma, her clinical presentation was mild bilateral sixth nerve palsy only

2 years. The other patient with metastatic lesion had been treated with surgical resection, chemotherapy, and radiotherapy for osteosarcoma in the right thigh before the development of sixth cranial nerve palsy. She was diagnosed as metastatic sixth cranial nerve palsy during treatment for metastasis to lumbar spine.

Among three patients with Miller Fisher syndrome, two patients had mild bilateral sixth cranial nerve palsy with small degree of esotropia and limitation of abduction of $\sim 10 \%$ of normal range. One of them had combined dizziness, vertigo, and dysarthria. One patient with Miller Fisher syndrome showed moderate limitation of abduction of $\sim 30-40 \%$ of normal range in one eye without limitation of ocular movement to other directions. The patient had recalled a similar experience 8 years ago that resolved spontaneously.

In six patients, non-aneurysmal vascular contact was the presumed cause of sixth cranial nerve palsy. Among these six patients, five showed partial or complete resolution of paralysis spontaneously. Trauma was the presumed cause of sixth cranial nerve palsy in another four patients, whereas the cause was undefined in another four patients. It was classified as idiopathic. Among these patients with undefined causes, one patient experienced two more episodes of unilateral sixth cranial nerve palsy 2 and 5 years later after the first sixth cranial nerve palsy preceded by flu symptoms in both recurred episodes. They spontaneously resolved within several months.

\section{Third cranial nerve palsy}

Of 14 subjects with third cranial nerve palsy, neuroimaging and other studies identified neoplasia involving central nervous system in four patients, including one patient with progression from previously diagnosed malignancy and one patient who underwent surgical removal of brain tumor. Both patients with newly diagnosed primary brain tumor had tumor associated with neurofibromatosis. One patient who had vascular malformation and intracranial hemorrhage had the first clinical presentation of isolated third cranial nerve palsy. Three patients had inflammation and one patient had intracranial hemorrhage without vascular malformation. In one patient, non-aneurysmal vascular contact was the presumed cause of third cranial nerve palsy. In another one patient, trauma was the presumed cause of third cranial nerve palsy. In three patients, the cause was not defined. It was classified as idiopathic.

\section{Fourth cranial nerve palsy}

Of 13 subjects with fourth cranial nerve palsy, neuroimaging and other studies identified neoplasia involving central nervous system in two patients, one of whom had schwannoma of the fourth nerve. This patient had negative conventional MRI results. When repeated high-resolution MRI was performed for this patient, neoplasia was found. The other patient had vascular malformation. One patient had hemorrhagic infarction after seizure. In another patient, trauma was the presumed cause of fourth cranial nerve palsy. In two patients, the cause was not defined (classified as idiopathic) (Fig. 2). The onset time of fourth cranial nerve palsy was determined based on reports obtained from patients and their guardians regarding head tilt supported by old photo and the onset of diplopia if present. However, the etiology was concluded as congenital after detailed investigation in six patients based on the absence or atrophy of fourth cranial nerve and/or superior oblique muscle in the paretic eye.

\section{Combined cranial nerve palsy}

For four patients with combined cranial nerve palsy, Miller Fisher syndrome was a causative diagnosis for three patients while cavernous sinus thrombophlebitis was diagnosed for one patient. 


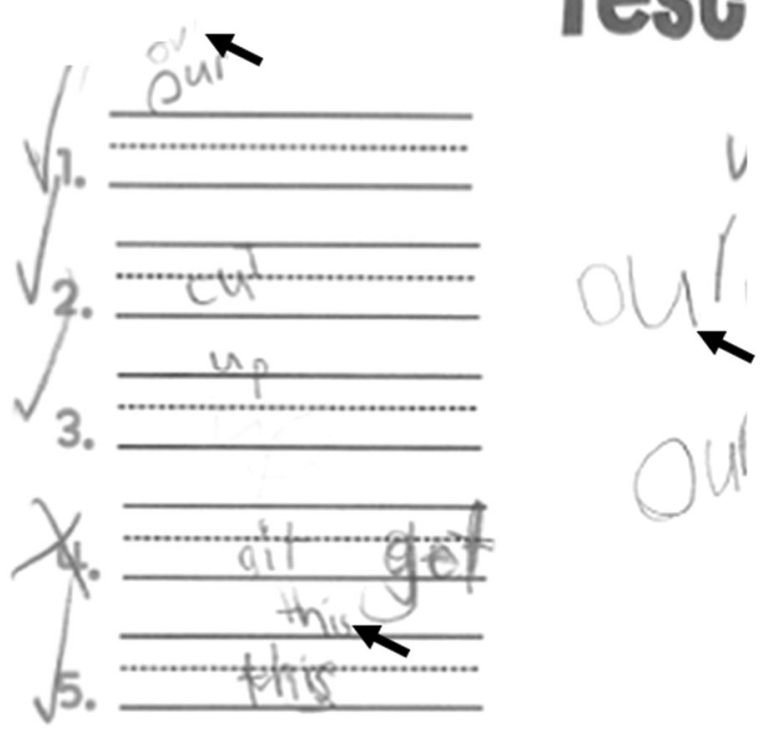

Fig. 2 A test sheet of a 5-year-old girl who presented with acute-onset double vision owing to acquired fourth nerve palsy. Observation of change in usual life including writing and head posture could be an important clue to provide medical attention to children with third, fourth, or sixth cranial nerve palsies. Her teacher in the kindergarten first noticed that the patient started to write sentences twice, one in the original line and one in the oblique to the original line (arrows). The patient stated that she suddenly started to see double images separated vertically and obliquely. She had acquired unilateral fourth nerve palsy. Through thorough work-up including full history taking, magnetic resonance imaging, and blood work, the patient was determined to be idiopathic. Her symptom and sign spontaneously resolved completely within 1 week

\section{Classification according to the presence of significant past medical history}

When patients were divided according to the presence of significant past medical history including preterm birth, malignancy, any neurologic, cardio-vascular, and systemic disorder, 18 patients were identified as having significant past medical history (Table 2). Among these 18 patients, the most common cause of cranial nerve palsy was neoplasia (44\%). Among 48 patients without significant past medical history, the most common causes of cranial nerve palsy were neoplasia (15\%), inflammation (15\%), and nonaneurysmal vascular contact $(15 \%)$.

\section{Classification according to isolated vs. non-isolated third, fourth, and sixth cranial nerve palsy}

Among 66 patients with third, fourth, or sixth cranial nerve palsies, 57 had neurologically isolated palsy (Table 3). They had no neurologic signs or symptoms except headache or periorbital pain within 1 month after the onset of diplopia and isolated third, fourth, or sixth cranial nerve palsies. Among nine patients with not-isolated third,
Table 2 The number of patients according to significant past medical history including preterm birth, malignancy, any neurologic, cardiovascular, and systemic disorder

\begin{tabular}{|c|c|c|}
\hline & \multicolumn{2}{|c|}{$\begin{array}{l}\text { Third, fourth, and sixth cranial } \\
\text { nerve palsies }\end{array}$} \\
\hline & $\begin{array}{l}\text { with PMHx } \\
(n=18)\end{array}$ & $\begin{array}{l}\text { without PMHx } \\
(n=48)\end{array}$ \\
\hline Age (mean $\pm \mathrm{SD})$ (years) & $8 \pm 7$ & $9 \pm 6$ \\
\hline Gender (male/female) & $13 / 5$ & $30 / 18$ \\
\hline \multicolumn{3}{|l|}{ Underlying etiologies } \\
\hline Neoplastic & $8(44 \%)$ & $7(15 \%)$ \\
\hline Gliomatous & & 4 \\
\hline Other primary & 3 & 3 \\
\hline Metastatic or progression & 3 & 0 \\
\hline $\begin{array}{l}\text { After surgical resection of brain } \\
\text { tumor }\end{array}$ & 2 & 0 \\
\hline Inflammatory & & $7(15 \%)$ \\
\hline Intracranial hemorrhage & $2(11 \%)$ & $1(2 \%)$ \\
\hline Cavernous sinus thrombophlebitis & & $1(2 \%)$ \\
\hline Vascular malformation & & $2(4 \%)$ \\
\hline Hydrocephalus & $2(11 \%)$ & \\
\hline Multiple sclerosis & & $1(2 \%)$ \\
\hline $\begin{array}{l}\text { Chemotherapy induced } \\
\text { leukoencephalopathy }\end{array}$ & $1(6 \%)$ & \\
\hline Non-aneurysmal vascular contact & & $7(15 \%)$ \\
\hline Miller Fisher syndrome & & $6(13 \%)$ \\
\hline Traumatic & & $6(13 \%)$ \\
\hline Idiopathic & $5(28 \%)$ & $4(8 \%)$ \\
\hline Congenital $^{\mathrm{a}}$ & & $6(13 \%)$ \\
\hline
\end{tabular}

$\mathrm{SD}=$ standard deviation; $\mathrm{PMHx}=$ past medical history including preterm birth, malignancy, any neurologic, cardio-vascular, or systemic disorder

${ }^{a}$ These patients had acquired onset. However, the etiology was concluded as congenital based on the absence or atrophy of fourth nerve and/or superior oblique muscle in the paretic eye

fourth, or sixth cranial nerve palsies, four had sixth cranial nerve palsy. Among them, two patients had combined papilledema owing to hydrocephalus, one patient had combined gait disturbance owing to brain tumor, and one patent had vertigo and dysarthria associated with Miller fisher syndrome. One patient had third cranial nerve palsy combined with hemianopia. Four patients had combined palsies of two to three nerves among third, fourth, and sixth cranial nerves. One of these patients had concomitant gait imbalance.

\section{Classification according to age}

Table 4 presents the number of patients according to the age of onset of third, fourth, and sixth cranial nerve palsies. Neoplasia involving central nervous system was one of the 
Table 3 The number of patients according to isolated vs. non-isolated third, fourth, and sixth cranial nerve palsies

\begin{tabular}{lll}
\hline & \multicolumn{2}{l}{$\begin{array}{l}\text { Third, fourth, and sixth cranial } \\
\text { nerve palsies }\end{array}$} \\
\cline { 2 - 3 } & Isolated $(n=57)^{\mathrm{a}}$ & $\begin{array}{l}\text { Not-isolated } \\
(n=9)\end{array}$ \\
\hline Age & $8 \pm 7$ & $11 \pm 5$ \\
Gender & $37 / 20$ & $6 / 3$ \\
Underlying etiologies & & \\
Neoplastic & $13(23 \%)$ & $2(22 \%)$ \\
$\quad$ Gliomatous & 4 & \\
Other primary & 6 & 1 \\
Metastatic or progression & 2 & 1 \\
After surgical resection of brain & 1 & \\
tumor & & \\
Inflammatory & $7(12 \%)$ & \\
Intracranial hemorrhage & $3(5 \%)$ & \\
Cavernous sinus thrombophlebitis & 0 & \\
Vascular malformation & $2(4 \%)$ & \\
Hydrocephalus & 0 & $1(22 \%)$ \\
Multiple sclerosis & $1(2 \%)$ & \\
Chemotherapy induced & $1(2 \%)$ & \\
leukoencephalopathy & $7(12 \%)$ & \\
Non-aneurysmal vascular contact & & \\
Miller Fisher syndrome & $2(4 \%)$ & \\
Traumatic & $6(11 \%)$ & \\
Idiopathic & & \\
Congenital & & \\
\hline
\end{tabular}

$\mathrm{SD}=$ standard deviation

${ }^{a}$ Neurologically isolated palsy means no neurologic sign or symptom other than isolated third, fourth, or sixth cranial nerve palsies except for headache or periorbital pain within 1 month after the onset of diplopia

These patients had acquired onset. However, the etiology was concluded as congenital based on the absence or atrophy of fourth nerve and/or superior oblique muscle in the paretic eye

most common causes of third, fourth, and sixth cranial nerve palsies in patients aged $0-14$ years or $15-19$ years.

\section{Discussion}

With the advent of modern neuroimaging, imaging techniques have been rapidly developed. Three-dimensional steady-state free precession sequences and modified fully refocused steady-state sequences such as CISS/FIESTA-C/ B-FFE especially can enhance display of each cranial nerve and enable more accurate diagnosis. Analyzing the cause of isolated third, fourth, and sixth cranial nerve palsies using these modern neuroimaging techniques with laboratory work-up could be valuable at present era.
Table 4 The number of patients according to the age of onset of third, fourth, and sixth cranial nerve palsies

\begin{tabular}{|c|c|c|}
\hline & \multicolumn{2}{|c|}{$\begin{array}{l}\text { Third, fourth, and sixth cranial } \\
\text { nerve palsies }\end{array}$} \\
\hline & $\begin{array}{l}\text { Age of onset } \\
0-14 \text { years } \\
(n=50)\end{array}$ & $\begin{array}{l}\text { Age of onset } \\
15-19 \text { years } \\
(n=16)\end{array}$ \\
\hline Age & $6 \pm 5$ & $17 \pm 2$ \\
\hline Gender & $34 / 16$ & $9 / 7$ \\
\hline \multicolumn{3}{|l|}{ Underlying etiologies } \\
\hline Neoplastic & $10(20 \%)$ & $5(31 \%)$ \\
\hline Gliomatous & 3 & 1 \\
\hline Other primary & 5 & 1 \\
\hline Metastatic or progression & 1 & 2 \\
\hline $\begin{array}{l}\text { After surgical resection of brain } \\
\text { tumor }\end{array}$ & 1 & 1 \\
\hline Inflammatory & $7(14 \%)$ & \\
\hline Intracranial hemorrhage & $3(6 \%)$ & \\
\hline $\begin{array}{l}\text { Cavernous sinus } \\
\text { thrombophlebitis }\end{array}$ & $1(2 \%)$ & \\
\hline Vascular malformation & $2(4 \%)$ & \\
\hline Hydrocephalus & $2(4 \%)$ & \\
\hline Multiple sclerosis & 0 & $1(6 \%)$ \\
\hline $\begin{array}{l}\text { Chemotherapy induced } \\
\text { leukoencephalopathy }\end{array}$ & $1(2 \%)$ & \\
\hline $\begin{array}{l}\text { Non-aneurysmal vascular } \\
\text { contact }\end{array}$ & $6(12 \%)$ & $1(6 \%)$ \\
\hline Miller Fisher syndrome & $3(6 \%)$ & $3(19 \%)$ \\
\hline Traumatic & $4(8 \%)$ & $2(13 \%)$ \\
\hline Idiopathic & $5(10 \%)$ & $4(25 \%)$ \\
\hline Congenital $^{\mathrm{a}}$ & $6(12 \%)$ & \\
\hline
\end{tabular}

$\mathrm{SD}=$ standard deviation

${ }^{\text {a }}$ These patients had acquired onset. However, the etiology was concluded as congenital based on the absence or atrophy of fourth nerve and/or superior oblique muscle in the paretic eye

\section{Neoplasia}

Our results showed that neoplasia involving central nervous system was one of the most common causes overall in patients with third, fourth, and sixth cranial nerve palsies in both age groups (0-14 years and 15-19 years). A total of $23 \%$ of patients with acute third, fourth, or sixth cranial nerve palsies were found to have neoplasia in this study. Neoplasia was the most common cause of both sixth and third cranial nerve palsies. The reported proportion of neoplasia in children with cranial nerve palsies varied between studies. It has been reported that $19-39 \%$ of children with sixth cranial nerve palsies have tumor as a main cause of nerve palsy $[2,3,6,10]$. Among children with third cranial nerve palsy, the proportion of tumor has been reported to be $6-14 \%[6,8,11]$. Kodsi et al. [6] have 
previously reported that neoplasia is a cause of palsy in 5\% of children with acquired fourth cranial nerve palsy. Direct comparison between reports was difficult because several studies were carried out before the routine use of MRI. In addition, study settings and population were different between studies. However, our study results, as well as previous studies, showed that substantial proportion of children with cranial nerve palsies had neoplasia as a causative lesion. In our study, 13 of 15 patients with neoplasia involving central nervous system $(87 \%)$ had isolated third, fourth, or sixth cranial nerve palsies as the presenting sign. In addition, two patients with negative conventional MRI results were found to have neoplasia when repeated highresolution MRI was performed. This suggests that highresolution contrast-enhanced MRI and repeated imaging are important for clinically suspected patients so that intracranial neoplasia will not be missed.

\section{Inflammation}

Overall, a total of $11 \%$ of pediatric patients with third, fourth, and sixth cranial nerve palsies showed inflammation in this study. Robertson et al. [10] have previously reported that inflammation was the third most common cause of acquired sixth nerve palsy in children, following neoplasia and trauma. Miller et al. [16] have also reported that 4 out of $30(13 \%)$ children with third nerve palsy have inflammation as a cause of palsy. One of them had bacterial meningitis. Another patient had a probable viral meningitis, whereas the other two patients were presumed to have post-viral inflammation [16].

\section{Idiopathic}

It was noteworthy that idiopathic cause of third, fourth, and sixth cranial nerve palsies (for which cause was not determined) was the second most common cause following neoplasia, especially in patients aged 15-19 years. All patients classified as idiopathic who underwent highresolution MRI with three-dimensional steady-state free precession sequences and modified fully refocused steadystate sequences (CISS) were also negative for serologic results, syphilis serologic results, thyroid-stimulating hormone receptor antibodies, acetylcholine receptor antibody, and ganglioside antibody panel. Three of them underwent edrophonium test, neostigmine test, and repetitive nerve stimulation test depending on their clinical presentation. For patients aged $15-19$ years and $0-14$ years, $25 \%$ and $10 \%$ of them, respectively, were categorized as idiopathic third, fourth, and sixth cranial nerve palsies. In previous studies, $5-17 \%$ of children with third, fourth, and sixth cranial nerve palsies had idiopathic cause $[2-4,6,7,10]$. Peters et al. [17] have previously reported that $13 \%$ of non-traumatic sixth cranial nerve palsy in young adults aged $20-50$ are idiopathic. In their study, when isolated sixth cranial nerve palsies were analyzed separately, the incidence of idiopathic sixth cranial nerve palsy was increased to $18 \%$ [17]. Five $(56 \%)$ of nine patients with idiopathic third, fourth, and sixth cranial nerve palsies in the present study had significant past medical history, including neurologic, cardiovascular, and systemic disorder. Patients who were classified as idiopathic might have other disorders including multiple sclerosis and myasthenia gravis with a longer follow-up.

\section{Others}

Two patients with hydrocephalus and sixth cranial nerve palsy had coexisting papilledema or optic atrophy. No patient had idiopathic intracranial hypertension in the present study. Lee et al. [7] have reported that idiopathic intracranial hypertension is a cause of sixth cranial nerve palsy in $8 \%$ of children in their study. The difference in the proportion of this etiology might be due to difference in the prevalence of idiopathic intracranial hypertension between races. It has been reported that the prevalence of idiopathic intracranial hypertension is much lower in Asia than that in Western countries [18].

Non-aneurysmal vascular contact was the presumed cause of sixth or third cranial nerve palsy in seven patients in this study. Non-aneurysmal neurovascular contact was rarely considered as a cause of third, fourth, or sixth palsy in the past because anatomic features were not readily demonstrable by conventional imaging studies. Several recent studies have reported that neurovascular contact is not a rare anatomic phenomenon in third or sixth cranial nerve $[19,20]$. Although neurovascular contact is not always pathogenic, previous studies have suggested that demonstration of neurovascular compression in patients with cranial nerve palsies could have diagnostic value $[15,19]$. It has been postulated that detection of neurovascular compression might have high sensitivity but low specificity for nerve palsy [21, 22]. True causative associations between non-aneurysmal vascular contact and various cranial nerve palsies should be discussed further. The significance and prognosis value of neurovascular compression in cranial nerve palsies merit further studies in the future.

Late decompensation in congenital fourth cranial nerve palsy was the most common cause of fourth cranial nerve palsy in the present study. For these patients, the etiology was concluded as congenital after detailed investigation based on the absence or atrophy of fourth never and/or superior oblique muscle in paretic eye, although the onset time was determined by patients' and guardians' report regarding head tilt and the onset of diplopia if present along 
with support from old photo. Lee et al. [23] previously reported two cases of trochlear nerve agenesis causing superior oblique palsy that emerged later in life. In their cases, congenital trochlear palsy owing to agenesis developed vertical diplopia after age 50 probably due to delayed decompensation.

In the present study, six patients were diagnosed as Miller Fisher syndrome based on ganglioside antibody testing. All of them showed positive results for anti-GQ1b antibody testing. Among these six patients, two initially presented as mild bilateral sixth cranial nerve palsy and one patient presented as unilateral sixth cranial nerve palsy other than combined oculomotor paralysis. Tatsumoto et al. [24] have previously reported a case of sixth cranial nerve paresis with paresthesias in a patient with positive result for anti-GQ1b antibody testing. Two cases with mild bilateral sixth cranial nerve palsy might have weakness in other extraocular muscles. However, such weakness was lower than the level of detection considering that the limitation of abduction was very mild $(\sim 10 \%$ of normal range in these cases). Based on our results, the possibility of Miller Fisher syndrome might be considered in any case of isolated third, fourth, or sixth cranial nerve palsy.

The overall proportion of trauma was $9 \%$ in children with ocular motor nerve palsies in this study. Such relatively low proportion of trauma in our study compared with that in previous studies might be owing to difference in study settings including population, referral pattern, and location of the hospital $[2,3,6,10,11]$.

\section{Limitations}

This study has several limitations. First, this study was retrospective in nature. The protocol of neuroimaging was not identical in all subjects because we conducted highresolution brain MRI to visualize cranial nerves only when the etiology could not be clearly revealed from previous brain images. Also, only selected patients underwent edrophonium test, neostigmine test, and repetitive nerve stimulation test depending on their clinical presentation. Some patients with myasthenia gravis without clinically suspected symptom or sign might have been missed. Second, there might be selection bias in this study. The source of patients came from patients who were referred to a tertiary hospital. This could increase the prevalence of serious underlying pathologies in our study. Owing to difference in referral patterns, the proportion of several specific etiologies associated with third, fourth, and sixth cranial nerve palsies such as trauma might be affected. Third, because all data were obtained from one ethnicity, direct application of these data to other races might be inappropriate.

\section{Conclusions}

In conclusion, a substantial proportion of pediatric and juvenile patients had serious pathologies of third, fourth, and sixth cranial nerve palsies such as neoplasia, inflammation, and hemorrhage involving the central nervous system. Overall, $23 \%$ of patients with acute third, fourth, or sixth cranial nerve palsies were found to have neoplasia in this study. Owing to life-threatening nature of possible pathologies, prompt and accurate diagnosis of etiologies using high-resolution MRI with contrast and proper laboratory work-up are important for pediatric and juvenile patients with cranial nerve palsies.

\section{Summary Table}

\section{What was known before}

- Neoplasm or trauma has been reported to be a major cause in pediatric population in third, fourth, or sixth cranial nerve palsies in studies using conventional MRI.

\section{What this study adds}

- Using high-resolution MRI with three-dimensional steady-state free precession sequences and modified fully refocused steady-state sequences (CISS) and detailed laboratory work-up, etiologies of cranial nerve palsies were analyzed. Overall, $23 \%$ of patients with acute third, fourth, or sixth cranial nerve palsies were found to have neoplasms in this study and confirmed previous results. We also analyzed the proportion of non-aneurysmal neurovascular contact and Miller Fisher syndrome among patients with third, fourth, and sixth cranial nerve palsies, using MRI with CISS and laboratory work-up.

Acknowledgements The authors have no proprietary or commercial interest in any materials discussed in this article. Kyung-Ah Park and Sei Yeul Oh (Sungkyunkwan University, Neuro-Ophthalmology) contributed to study concept and design. Kyung-Ah Park and Sei Yeul Oh (Sungkyunkwan University, Neuro-Ophthalmology) and Ju-Hong Min and Byoung Joon Kim (Sungkyunkwan University, Neurology) were involved in data collection. Kyung-Ah Park (Sungkyunkwan University, Neuro-Ophthalmology), Ju-Hong Min and Byoung Joon Kim (Sungkyunkwan University, Neurology) contributed to data analysis and data interpretation. Sei Yeul Oh (Sungkyunkwan University, Neuro-Ophthalmology), Ju-Hong Min, Byoung Joon Kim (Sungkyunkwan University, Neurology), and Yikyung Kim (Sungkyunkwan University, Radiology) were involved in critical revision of manuscript for intellectual content.

\section{Compliance with ethical standards}

Conflict of interest The authors declare that they have no conflict of interest. 
Publisher's note: Springer Nature remains neutral with regard to jurisdictional claims in published maps and institutional affiliations.

\section{References}

1. Holmes JM, Mutyala S, Maus TL, Grill R, Hodge DO, Gray DT. Pediatric third, fourth, and sixth nerve palsies: a population-based study. Am J Ophthalmol. 1999;127:388-92.

2. Afifi AK, Bell WE, Menezes AH. Etiology of lateral rectus palsy in infancy and childhood. J Child Neurol. 1992;7:295-9.

3. Aroichane M, Repka MX. Outcome of sixth nerve palsy or paresis in young children. J Pediatr Ophthalmol Strabismus. 1995;32:152-6.

4. Harley RD. Paralytic strabismus in children. Etiologic incidence and management of the third, fourth, and sixth nerve palsies. Ophthalmol. 1980;87:24-43.

5. Keith CG. Oculomotor nerve palsy in childhood. Aust N Z J Ophthalmol. 1987;15:181-4.

6. Kodsi SR, Younge BR. Acquired oculomotor, trochlear, and abducent cranial nerve palsies in pediatric patients. Am J Ophthalmol. 1992;114:568-74.

7. Lee MS, Galetta SL, Volpe NJ, Liu GT. Sixth nerve palsies in children. Pediatr Neurol. 1999;20:49-52.

8. Miller NR. Solitary oculomotor nerve palsy in childhood. Am J Ophthalmol. 1977;83:106-11.

9. Ng YS, Lyons CJ. Oculomotor nerve palsy in childhood. Can J Ophthalmol. 2005;40:645-53.

10. Robertson DM, Hines JD, Rucker CW. Acquired sixth-nerve paresis in children. Arch Ophthalmol. 1970;83:574-9.

11. Schumacher-Feero LA, Yoo KW, Solari FM, Biglan AW. Third cranial nerve palsy in children. Am J Ophthalmol. 1999; 128:216-21.

12. Chavhan GB, Babyn PS, Jankharia BG, Cheng HL, Shroff MM. Steady-state MR imaging sequences: physics, classification, and clinical applications. Radiographics. 2008;28:1147-60.

13. Sheth S, BFt Branstetter, Escott. EJ. Appearance of normal cranial nerves on steady-state free precession MR images. Radiographics. 2009;29:1045-55.
14. Joshi S, Tee WWH, Franconi C, Prentice D. Transient oculomotor nerve palsy due to non-aneurysmal neurovascular compression. $\mathrm{J}$ Clin Neurosci. 2017;45:136-7.

15. Tsai TH, Demer JL. Nonaneurysmal cranial nerve compression as cause of neuropathic strabismus: evidence from high-resolution magnetic resonance imaging. Am J Ophthalmol. 2011;152:1067-. e1062.

16. Miller RW, Lee AG, Schiffman JS, Prager TC, Garza R, Jenkins PF, et al. A practice pathway for the initial diagnostic evaluation of isolated sixth cranial nerve palsies. Med Decis Mak. 1999;19:42-48.

17. Peters GB3rd, Bakri SJ, Krohel GB. Cause and prognosis of nontraumatic sixth nerve palsies in young adults. Ophthalmology. 2002;109:1925-8.

18. Yabe I, Moriwaka F, Notoya A, Ohtaki M, Tashiro K. Incidence of idiopathic intracranial hypertension in Hokkaido, the northernmost island of Japan. J Neurol. 2000;247:474-5.

19. Liang C, Du Y, Lin X, Wu L, Wu D, Wang X. Anatomical features of the cisternal segment of the oculomotor nerve: neurovascular relationships and abnormal compression on magnetic resonance imaging. J Neurosurg. 2009;111:1193-1200.

20. Yousry I, Camelio S, Wiesmann M, Schmid UD, Moriggl B, Bruckmann $\mathrm{H}$, et al. Detailed magnetic resonance imaging anatomy of the cisternal segment of the abducent nerve: Dorello's canal and neurovascular relationships and landmarks. J Neurosurg. 1999;91:276-83.

21. Boecher-Schwarz HG, Bruehl K, Kessel G, Guenthner M, Perneczky A, Stoeter P. Sensitivity and specificity of MRA in the diagnosis of neurovascular compression in patients with trigeminal neuralgia. A correlation of MRA and surgical findings. Neuroradiology. 1998;40:88-95.

22. Du C, Korogi Y, Nagahiro S, Sakamoto Y, Takada A, Ushio Y, et al. Hemifacial spasm: three-dimensional MR images in the evaluation of neurovascular compression. Radiology. 1995;197:227-31.

23. Lee S, Kim SH, Yang HK, Hwang JM, Kim JH, Kim JS, et al. Imaging demonstration of trochlear nerve agenesis in superior oblique palsy emerging during the later life. Clin Neurol Neurosurg. 2015;139:269-71.

24. Tatsumoto M, Odaka M, Hirata K, Yuki N. Isolated abducens nerve palsy as a regional variant of Guillain-Barre syndrome. J Neurol Sci. 2006;243:35-38. 